\title{
Atypically small temperature-dependence of the direct band gap in the metastable semiconductor copper nitride $\mathrm{Cu}_{3} \mathrm{~N}$
}

\author{
Max Birkett, ${ }^{1, *}$ Christopher N. Savory, ${ }^{2}$ Angela N. Fioretti, ${ }^{3}$ Paul Thompson, ${ }^{4}$ Christopher A. Muryn, ${ }^{5}$ A. D. Weerakkody, ${ }^{6}$ \\ I. Z. Mitrovic, ${ }^{6}$ S. Hall, ${ }^{6}$ Rob Treharne, ${ }^{1}$ Vin R. Dhanak, ${ }^{1}$ David O. Scanlon, ${ }^{2,7}$ Andriy Zakutayev, ${ }^{3}$ and Tim D. Veal ${ }^{1, \dagger}$ \\ ${ }^{1}$ Stephenson Institute for Renewable Energy and Department of Physics, University of Liverpool, Liverpool L69 7ZF, United Kingdom \\ ${ }^{2}$ University College London, Kathleen Lonsdale Materials Chemistry, Department of Chemistry, 20 Gordon Street, \\ London WC1H OAJ, United Kingdom \\ ${ }^{3}$ National Renewable Energy Laboratory, Denver West Parkway, Golden, Colorado 80401, USA \\ ${ }^{4} X M a S$, The UK CRG, European Synchrotron Radiation Facility, CS 40220, 38043 Grenoble Cedex 9, France \\ ${ }^{5}$ School of Chemistry and PSI, University of Manchester, Oxford Road, Manchester M13 9PL, United Kingdom \\ ${ }^{6}$ Department of Electrical Engineering and Electronics, University of Liverpool, Brownlow Hill, Liverpool L69 3GJ, United Kingdom \\ ${ }^{7}$ Diamond Light Source Ltd., Diamond House, Harwell Science and Innovation Campus, Didcot OX11 ODE, United Kingdom
}

(Received 18 November 2016; revised manuscript received 6 February 2017; published 6 March 2017)

\begin{abstract}
The temperature-dependence of the direct band gap and thermal expansion in the metastable anti- $\mathrm{ReO}_{3}$ semiconductor $\mathrm{Cu}_{3} \mathrm{~N}$ are investigated between 4.2 and $300 \mathrm{~K}$ by Fourier-transform infrared spectroscopy and $\mathrm{x}$-ray diffraction. Complementary refractive index spectra are determined by spectroscopic ellipsometry at $300 \mathrm{~K}$. A direct gap of $1.68 \mathrm{eV}$ is associated with the absorption onset at $300 \mathrm{~K}$, which strengthens continuously and reaches a magnitude of $3.5 \times 10^{5} \mathrm{~cm}^{-1}$ at $2.7 \mathrm{eV}$, suggesting potential for photovoltaic applications. Notably, the direct gap redshifts by just $24 \mathrm{meV}$ between 4.2 and $300 \mathrm{~K}$, giving an atypically small band-gap temperature coefficient $d E_{\mathrm{g}} / d T$ of $-0.082 \mathrm{meV} / \mathrm{K}$. Additionally, the band structure, dielectric function, phonon dispersion, linear expansion, and heat capacity are calculated using density functional theory; remarkable similarities between the experimental and calculated refractive index spectra support the accuracy of these calculations, which indicate beneficially low hole effective masses and potential negative thermal expansion below $50 \mathrm{~K}$. To assess the lattice expansion contribution to the band-gap temperature-dependence, a quasiharmonic model fit to the observed lattice contraction finds a monotonically decreasing linear expansion (descending past $10^{-6} \mathrm{~K}^{-1}$ below $80 \mathrm{~K}$ ), while estimating the Debye temperature, lattice heat capacity, and Grüneisen parameter. Accounting for lattice and electron-phonon contributions to the observed band-gap evolution suggests average phonon energies that are qualitatively consistent with predicted maxima in the phonon density of states. As band-edge temperature-dependence has significant consequences for device performance, copper nitride should be well suited for applications that require a largely temperature-invariant band gap.
\end{abstract}

DOI: 10.1103/PhysRevB.95.115201

\section{INTRODUCTION}

Copper nitride $\mathrm{Cu}_{3} \mathrm{~N}$ is a relatively unexplored metastable semiconductor that has been studied for potential applications in write-once optical data storage [1-3], resistive random access memories [4], hybrid organic-inorganic solar cells [5], magnetic nanostructures, spin barrier tunnel junctions [6], and for rendering conductive dots and lines by maskless laser or electron-beam writing [7-9]. Applications may exploit the low decomposition temperature of $\sim 300^{\circ} \mathrm{C}$, above which $\mathrm{Cu}_{3} \mathrm{~N}$ decomposes to metallic copper and $\mathrm{N}_{2}$. Lately, $\mathrm{Cu}_{3} \mathrm{~N}$ has attracted interest as a candidate nontoxic, earth-abundant absorber for thin-film photovoltaics [10]. Favorable characteristics for photovoltaics include a beneficial band structure for AM1.5 solar illumination; strong above-onset absorption; material that is dopable both $p$ and $n$ type [11,12]: suggesting

\footnotetext{
*max.birkett@gmail.com

†T.Veal@liverpool.ac.uk
}

Published by the American Physical Society under the terms of the Creative Commons Attribution 4.0 International license. Further distribution of this work must maintain attribution to the author(s) and the published article's title, journal citation, and DOI. the potential realization of $p n$ homojunctions; defect tolerance, and a surface which may be passivated by a native oxide; as well as good material stability and established polycrystalline growth routes $[10,13]$.

$\mathrm{Cu}_{3} \mathrm{~N}$ crystallizes in a rather open, cubic anti- $\mathrm{ReO}_{3}$ structure (space group $P m \overline{3} m$, number 221 , first determined by Juza and Hahn [14]), comprising a cubic network of vertex-connected $\mathrm{NCu}_{6}$ octahedra. The structure is rather similar to the antiperovskite structure $\mathrm{ABX}_{3}$ (where $A$ and $B$ are anions and $X$ is a cation) without the $A$ anion in the $\frac{1}{2} \frac{1}{2} \frac{1}{2}$ body-center position (Wyckoff $b$ position). The space vacated leaves substantial room to accommodate structural distortions or impurities. Some spread is apparent in various physical properties reported for $\mathrm{Cu}_{3} \mathrm{~N}$, as illustrated by the lattice parameter and band gap which range from 3.807(4) [14] through $3.89 \AA$ [15] and 0.25 to $1.9 \mathrm{eV} \mathrm{[16],} \mathrm{respectively.}$ Such variability is thought to result from differences in nitrogen content, which may vary at growth time due to nitrogen liberation [16] and is implicated in affecting the stoichiometry [17], band gap [18], native acceptor concentration [19], conductivity [20], preferential orientation [17] and decomposition temperature [16]. Stoichiometric, copper-, or nitrogen-rich films may all be prepared, with excess copper or nitrogen atoms likely incorporated in the body-center position 
or at grain boundaries [15,21]. Preferential orientation is often seen in X-ray diffraction of $\mathrm{Cu}_{3} \mathrm{~N}$ films [10,22], with (111)- and (100)-oriented films prepared at respectively lower and higher substrate temperatures [23]. Because (111) planes comprise only nitrogen atoms, while (100) planes feature predominantly copper atoms, liberation of the predominant atoms from the surface leaves (111)- and (100)-oriented films which are respectively $\mathrm{Cu}$ and $\mathrm{N}$ rich [16,17,24]. As a metastable compound, $\mathrm{Cu}_{3} \mathrm{~N}$ is less stable than metallic copper and molecular $\mathrm{N}_{2}$; however, this results from the unusual strength of the $\mathrm{N}-\mathrm{N}$ bond: the metal-nitride bond is in fact just as strong as the metal-oxide bond [25].

The temperature-dependence of bands in semiconductors arises from the thermal expansion of the lattice, the effects of the electron-phonon interaction, and any temperaturedependent structural phase transitions. The temperature evolution of band offsets and effective masses may have significant implications for technological applications. In photovoltaics, efficiencies may fall by as much as $0.1 \% \mathrm{~K}^{-1}$, primarily due to increasing reverse saturation current and band-gap reduction at higher temperatures [26,27]. Despite the potential for temperature-dependent reconfiguration of the $\mathrm{Cu}_{3} \mathrm{~N}$ structure, no temperature-dependent structural phase transitions have yet been reported. A linear thermal expansion coefficient of $6.4(3) \times 10^{-6} \mathrm{~K}^{-1}$ was found between 20 and $300 \mathrm{~K}$ in $\mathrm{X}$-ray diffraction (XRD) of microcrystalline $\mathrm{Cu}_{3} \mathrm{~N}$ material [28] at atmospheric pressure; in contrast, $\mathrm{XRD}$ of $\mathrm{Cu}_{3} \mathrm{~N}$ single crystals at 130 and $294 \mathrm{~K}$ found no significant changes in the 3.819(1) $\AA$ lattice parameter [29] but suggested highly anisotropic copper displacements and a minor distortion from the anti- $\mathrm{ReO}_{3}$ structure: with the $\mathrm{N}-\mathrm{Cu}-\mathrm{N}$ bond angle relaxing from $173^{\circ}$ at $294 \mathrm{~K}$ to $176^{\circ}$ at $130 \mathrm{~K}$, explained as due to decreased mobility of the rigid $\mathrm{NCu}_{6}$ octahedra at the lower temperature. However, while accepting copper anisotropy and some disorder, static octahedral distortions were rejected in higher-precision neutron-diffraction studies [30]. Elsewhere, extended $\mathrm{x}$-ray absorption fine structure (EXAFS) between 10 and $300 \mathrm{~K}$ set an upper limit of $0.005 \AA$ on any elongation of the $\mathrm{Cu}-\mathrm{N}$ and $\mathrm{Cu}-\mathrm{Cu}$ bonds [31], consistent with the $0.0037 \AA$ elongation expected from linear expansion [28]. These works leave some questions on the possibility of structural distortions and the magnitude of thermal expansion in $\mathrm{Cu}_{3} \mathrm{~N}$. This work reports atypically small temperature-dependence of the direct band gap in $\mathrm{Cu}_{3} \mathrm{~N}$. The thermal expansion contribution to the band-gap temperature-dependence (assessed via XRD and density functional theory) suggests that the electron-phonon interaction lowers the band gap by 160 to $200 \mathrm{meV}$ between 4.2 and $300 \mathrm{~K}$, respectively.

\section{METHODS}

Polycrystalline $p$-type $\mathrm{Cu}_{3} \mathrm{~N}$ films $\left(p \sim 10^{16}-10^{17} \mathrm{~cm}^{-1}\right)$ were deposited on glass substrates (Corning Eagle XG, an alkaline-earth boro-aluminosilicate glass [32]) by reactive rf magnetron sputtering, with argon working gas and a rf plasma atomic nitrogen source (see also Refs. [10] and [12]). Temperature-dependent $\mathrm{x}$-ray diffraction was performed at five temperatures between 100 and $280 \mathrm{~K}$ using a Supernova X-ray diffractometer (Rigaku Oxford Diffraction) with a monochromated molybdenum anode $\left(\lambda_{K \alpha 1}\right.$ and $\lambda_{K \alpha 2}$ at 0.709317 and $0.713607 \AA$ ) [33] and an Eos CCD detector, and separately at three temperatures between 4.2 and $100 \mathrm{~K}$ using the XMaS laboratory source (ESRF, Grenoble, France) with a monochromated copper anode $\left(\lambda_{K \alpha 1}\right.$ and $\lambda_{K \alpha 2}$ at 1.540593 and $1.544427 \AA$ ) [33] and a point detector (avalanche photodiode). Infrared transmission and specular reflection spectroscopy was performed at $11^{\circ}$ angle of incidence for 17 temperatures between 4.2 and $300 \mathrm{~K}$ and photon energies of 0.37 to $3.1 \mathrm{eV}$ (3.3 to $0.4 \mu \mathrm{m}$ ) using a Bruker Vertex $70 \mathrm{v}$ Fourier-transform infrared (FTIR) spectrometer equipped with a combined reflection-transmission accessory and an Oxford Instruments CFV2 continuous-flow helium cryostat. To complement the FTIR absorption spectra, the $\mathrm{Cu}_{3} \mathrm{~N}$ dielectric function was evaluated separately with a Woollam M2000UI (rotating compensator) spectroscopic ellipsometer between 0.7 to $5.1 \mathrm{eV}$ (245 to $1690 \mathrm{~nm}$ ) at $300 \mathrm{~K}$ using incident angles of $65^{\circ}, 70^{\circ}$, and $75^{\circ}$. Scanning electron microscopy (SEM) indicated a film thickness of 0.98(9) $\mu \mathrm{m}$. Energy dispersive spectroscopy (EDS) using an Oxford Inca X-Act silicon drift detector found no impurity lines.

First-principles periodic density functional theory (DFT) calculations were performed through the Vienna $A b$ Initio Simulation Package (VASP) [34-37] using the screened HSE06 hybrid density functional [38-40] with a plane-wave basis set. Core and valence electron interactions were described by the projector augmented wave (PAW) method with $\mathrm{Cu}-3 d$ electrons treated as valence $[41,42]$. HSE06 was used for geometry optimization and band-structure calculations using a planewave cutoff energy of $560 \mathrm{eV}$, a $\Gamma$-centered $7 \times 7 \times 7 k$-point mesh and a geometry convergence criterion of $0.01 \mathrm{eV} / \AA$. The phonon dispersion curve, density of states, and lattice dynamics were evaluated in the Phonopy package [43,44] (with VASP) using a finite-displacement method with a 256 atom, $4 \times 4 \times 4$ supercell. Quasiharmonic calculations were performed on a range of unit cells, with cell volumes ranging $\pm 5 \%$ around the relaxed volume in intervals of $0.5 \%$, with a $60 \times 60 \times 60$ $\Gamma$-centered $q$ mesh used to sample thermodynamic properties. All phonon calculations used the PBEsol functional $[45,46]$, which has previously determined phonon behavior accurately in perovskite semiconductors [47]. Calculations giving the high-frequency dielectric function (and absorption spectrum) were performed using the method of Gajdos et al. [48].

\section{OPTICAL STUDIES}

The FTIR reflectivity $R_{t}(\hbar \omega)$ and transmissivity $T_{t}(\hbar \omega)$ were reduced to absorption spectra $\alpha(\hbar \omega)$ using equations which describe the reflection and transmission of light directed at normal incidence at an incoherent single optical layer of thickness $d$ with parallel faces

$$
\begin{aligned}
& R_{t}=R\left[1+\frac{(1-R)^{2} \exp (-2 \alpha d)}{1-R^{2} \exp (-2 \alpha d)}\right], \\
& T_{t}=\frac{(1-R)^{2} \exp (-\alpha d)}{1-R^{2} \exp (-2 \alpha d)},
\end{aligned}
$$

where $R(\hbar \omega)$ is the (unmeasured) intrinsic reflectivity at a semi-infinite boundary between the vacuum and the $\mathrm{Cu}_{3} \mathrm{~N}$ film (in contrast, the total reflectivity $R_{t}$ includes the contributions 


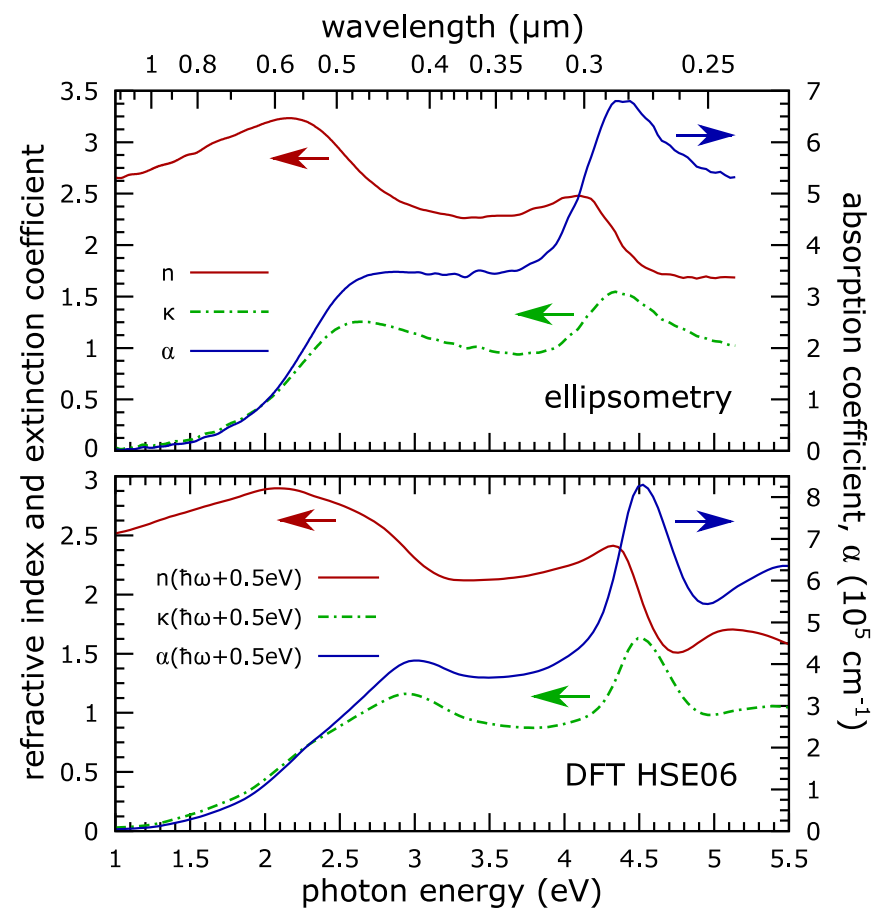

FIG. 1. Absorption and refractive index $(\tilde{n}=n-i \kappa)$ spectra via spectroscopic ellipsometry (at $300 \mathrm{~K}$ ) and HSE06 density functional theory. The HSE06 spectra are shifted by $0.5 \mathrm{eV}$ to lower photon energies, revealing impressive similarities with experiment. To recover the as-calculated HSE06 spectra (with $E_{\mathrm{VBM}}=0 \mathrm{eV}$ ), simply add $0.5 \mathrm{eV}$ to the abscissa axis labels.

from internal reflections at each face). The intrinsic reflectivity $R$ must be determined before proceeding; this is achieved using a self-consistent procedure: see Sec. 4 in the Supplemental Material [49].

Absorption and refractive index spectra determined by spectroscopic ellipsometry (SE) are shown in Fig. 1. Corresponding FTIR absorption spectra evaluated from equations (1) with a $1 \mu \mathrm{m}$ film thickness (as indicated by SEM and ellipsometry) are presented in Fig. 2(a). The SE and FTIR spectra are consistent below $1.8 \mathrm{eV}$, but some differences present above $1.8 \mathrm{eV}$, where very strong absorption leads to feeble transmission and so noise in the FTIR spectra of Fig. 2(a).

Visual inspection of either Fig. 1 or 2(a) suggests a strong absorption onset near $1.5 \mathrm{eV}$, possibly located above a weaker onset at $1.0 \mathrm{eV}$. Such a configuration would be consistent with the respective positions of the calculated [13] direct and indirect band gaps in $\mathrm{Cu}_{3} \mathrm{~N}$. In the ellipsometric spectra of Fig. 1, the absorption strength grows strongly to a local maximum of $3.5 \times 10^{5} \mathrm{~cm}^{-1}$ at $2.7 \mathrm{eV}$, maintaining this strength until increasing again at $3.7 \mathrm{eV}$. The strong absorption from $1.5 \mathrm{eV}$ supports the potential for $\mathrm{Cu}_{3} \mathrm{~N}$ as a candidate photovoltaic absorber. The edges of Fig. 2(a) show rather weak temperature dependence, with the band gap decreasing by some $32 \mathrm{meV}$ as the temperature is increased from 4.2 to $300 \mathrm{~K}$. Residual oscillations due to internal reflections are seen below $1.5 \mathrm{eV}$ in Fig. 2(a), as the incoherent model of Eq. (1) neglects interference effects.
The absorption spectra $\alpha(\hbar \omega)$ of Fig. 2(a) are sufficiently featureless that it is not possible to confidently discern separate regions for fitting combined direct and indirect onsets. Given that the direct onset should in any case be orders of magnitude stronger, the temperature-dependent spectra are fit with equations describing such an onset at a $M_{0}$ critical point [51]:

$$
\alpha_{\mathrm{d}} \hbar \omega=A \sqrt{R\left(\hbar \omega-E_{\mathrm{g}}^{\mathrm{d}}\right)},
$$

where $R(x)$ is the ramp function, $R(x)=x$ for $x \geqslant 0$ and $R(x)=0$ for $x<0$. Each direct onset includes an exponential Urbach tail which intrudes into the band gap,

$$
\alpha_{\mathrm{U}}(\hbar \omega)=B \exp \left[\gamma\left(\hbar \omega-E_{\mathrm{g}}^{\mathrm{d}}-E_{\mathrm{U}}\right)\right],
$$

where $E_{\mathrm{U}}$ is a material-dependent parameter that sets the point where the Urbach tail (3) smoothly and continuously joins the absorption edge of Eq. (2), and $B$ and $\gamma$ are functions only of $A$ [the same constant used in Eq. (2)], $E_{\mathrm{g}}^{\mathrm{d}}$ and $E_{\mathrm{U}}$ (see Supplemental Material Sec. 5 [49]). Combining these relations, the absorption associated with a direct onset is

$$
\alpha_{\text {direct }}(\hbar \omega)= \begin{cases}\alpha_{\mathrm{U}}(\hbar \omega), & \hbar \omega \leqslant\left(E_{\mathrm{g}}^{\mathrm{d}}+E_{\mathrm{U}}\right) \\ \alpha_{\mathrm{d}}(\hbar \omega), & \text { otherwise. }\end{cases}
$$

The results of fitting Eq. (4) using the LevenbergMarquardt algorithm are presented in Fig. 2(b). Sample fits and residuals are presented in the Supplemental Material Fig. 3 [49]. A direct band gap of $1.68 \mathrm{eV}$ is found at $300 \mathrm{~K}$, with a $24 \mathrm{meV}$ redshift seen between 4.2 and $300 \mathrm{~K}$, giving a band-gap temperature coefficient $d E_{\mathrm{g}} / d T$ of just $-0.082 \mathrm{meV} / \mathrm{K}$, rather smaller than seen typically in semiconductors [52]; in contrast, a comparison of twelve common semiconductors [53] including Si, Ge, GaAs, GaP, and CdTe showed mean (and standard deviation) band-gap temperature coefficients of $-0.36(7) \mathrm{meV} / \mathrm{K}$. The Urbach intercept $E_{\mathrm{U}}$ increases with temperature from 105 to $118 \mathrm{meV}$, see the inset in Fig. 2(b), while the absorption strength $A$ shows a scatter below $2 \%$ around $9 \times 10^{8} \mathrm{eV}^{0.5} \mathrm{~cm}^{-1}$.

The temperature-dependence of the optically determined band gaps of Fig. 2(b) is examined by fitting with the empirical Varshni relation [54],

$$
E_{\mathrm{g}}^{\mathrm{Varshni}}=E_{0}-\frac{\alpha T^{2}}{T+\beta},
$$

and with models comprising one and two Bose-Einstein oscillators [55-57],

$$
E_{\mathrm{g}}^{B E}=E_{0}+\sum_{i} \alpha_{i}\left[1+\frac{2}{\exp \left(\frac{E_{i}}{k_{\mathrm{B}} T}\right)-1}\right] .
$$

The results of the least-squares fits are shown as the solid and broken lines in Fig. 2(b). The Varshni fit suggests respective $E_{0}, \alpha$, and $\beta$ parameters of $1.70 \mathrm{eV}, 0.13 \mathrm{meV} / \mathrm{K}$ and $0.014 \mathrm{~K}$; such values are well within the typical range seen for Varshni parameters: as seen by respective mean (and standard deviation) $\alpha$ and $\beta$ values of $0.6(5) \mathrm{meV} / \mathrm{K}$ and $400(500) \mathrm{K}$ for fits to 37 common semiconductors [54,58]. The single Bose-Einstein oscillator fit finds a parameter $E_{0}$ of $1.70 \mathrm{eV}$ with respective average phonon and electron-phonon interaction energies $\left(E_{i}\right.$ and $\left.\alpha_{i}\right)$ of 0.846 and $-0.627 \mathrm{meV}$. The evolution seen in Fig. 2(b) differs somewhat from the growing band-gap reduction typically seen in semiconductors with 

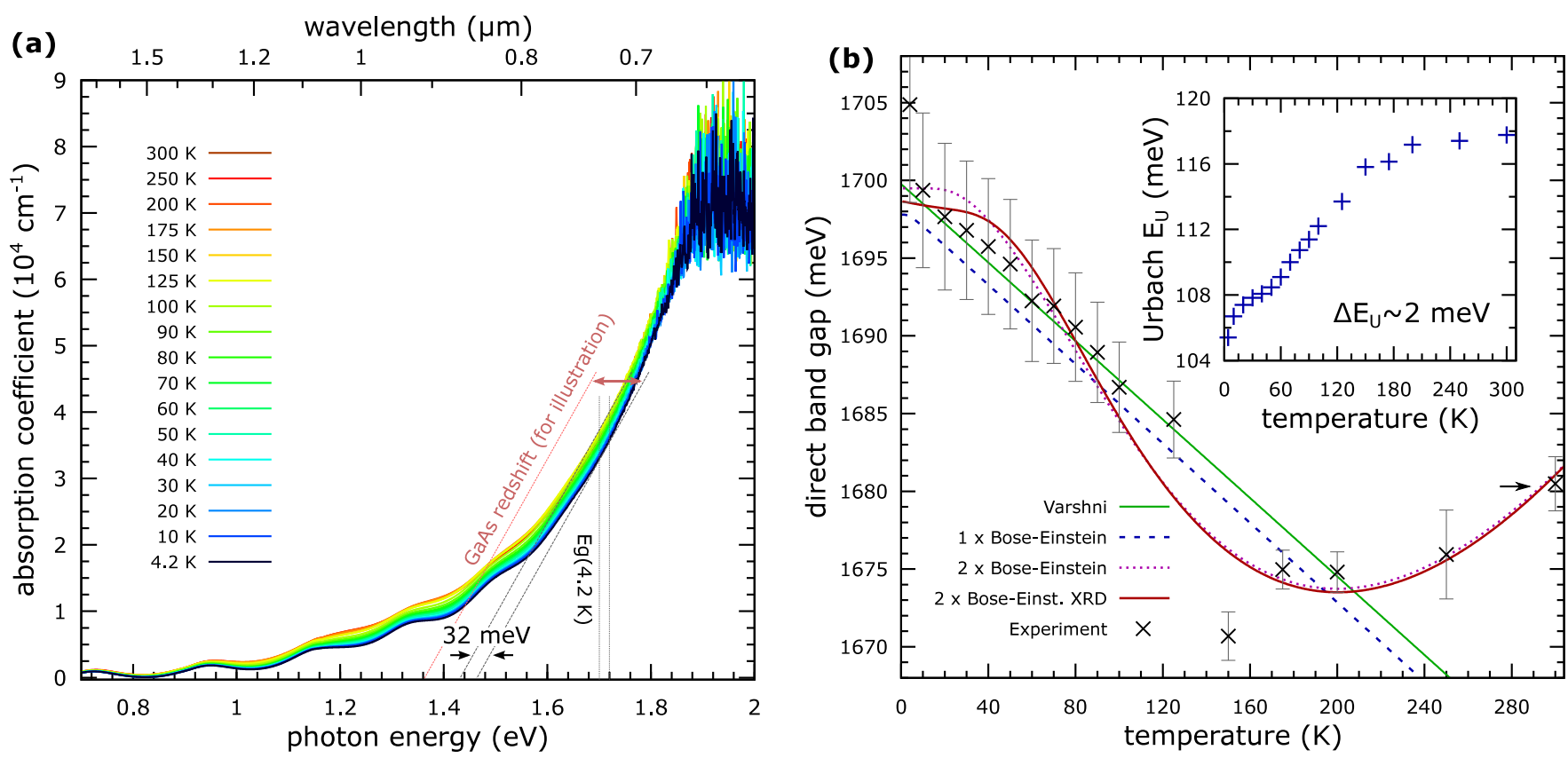

FIG. 2. Temperature-dependent absorption spectra fit as transitions at a direct gap ( $M_{0}$ critical point). (a) FTIR absorption spectra between 4.2 and $300 \mathrm{~K}$, over which the absorption edge shifts by some $32 \mathrm{meV}$ (compared with the $94 \mathrm{meV}$ of GaAs) [50]. (b) The temperature evolution of the $\mathrm{Cu}_{3} \mathrm{~N}$ band gap (as fit to the absorption edge) fit with the Varshni relation, single and double Bose-Einstein oscillators, and via a model which combines the double Bose-Einstein oscillator with the HSE06 band-gap dependence given by thermal lattice expansion (labeled Bose-Einst. XRD). The arrowed point is not fit via the Varshni relation or single Bose-Einstein oscillator. The inset shows the evolution of the Urbach energy $E_{\mathrm{U}}$ (with error $\Delta E_{\mathrm{U}}$ ).

increasing temperature $[52,55]$. Noting this, the fit statistics for the Varshni and single Bose-Einstein oscillator models deteriorate significantly with the $300 \mathrm{~K}$ data point. Admitting the possibility of an unidentified contributive factor, the $300 \mathrm{~K}$ data point was omitted for these fits only.

The physical model might be expected to improve with two oscillators because these cater to the different energy scales expected for acoustic and optical phonons. The double Bose-Einstein oscillator fit suggests an $E_{0}$ of $1.54(13) \mathrm{eV}$, and respective average phonon and interaction energies $\left\{E_{i}, \alpha_{i}\right\}$ of $\{10(6),-17(40)\}$ and $\{52(24), 180(100)\} \mathrm{meV}$. The mix of phonon energies is much improved with small and large phonon energies of reasonable magnitudes. Prior phonon calculations and experimental work [59], and the dispersion relations of Figs. 3(a) and 3(b) to be discussed shortly, suggest two threefold degenerate, zone-center $\Gamma_{15}$ IR-active modes, with energies of 11 and $78 \mathrm{meV}$. The inset of Supplemental Material Fig. 3 [49] shows a temperature-dependent feature at $78 \mathrm{meV}$ in the reflectivity and transmissivity spectra which is distinct from the substrate spectra. This feature is tentatively associated with the upper $\Gamma_{15}$ zone-center mode. The average phonon energies for the double Bose-Einstein oscillator appear rather reasonable upon review of the dispersion in Fig. 3(b): the lower and upper phonon energies accounting for maxima in the phonon density of states at 10 and $75 \mathrm{meV}$.

An interesting fit to Fig. 2(b) results by considering phenomena which contribute to the experimental band gap. The temperature-dependence of a band gap depends both on the lattice parameter and on electron-phonon and electron-hole interactions, which are usually omitted in DFT calculations. As the temperature-dependence of the lattice parameter is fully revealed by XRD, a DFT calculation at each lattice parameter then gives the thermal expansion contribution to the band-gap temperature-dependence. Differences between the experimental and DFT band gaps may then be attributed to phonon and electron-hole phenomena (or inaccuracies in the DFT gap). Writing the direct gap at any temperature $T$ as the sum of the DFT direct gap and a dual Bose-Einstein oscillator (representing phonon contributions) gives [60]

$$
\begin{aligned}
E_{\mathrm{g}}^{\text {direct }}(T) & =E_{\mathrm{g}}^{\text {thermal exp. }}(T)+E_{\mathrm{g}}^{\text {electron-phonon }}(T) \\
& =E_{\mathrm{g}}^{\text {XRD,DFT }}(T)+E_{\mathrm{g}}^{B E}(T) .
\end{aligned}
$$

Fitting with the gap $E_{\mathrm{g}}^{\mathrm{XRD}, \mathrm{HSEO}}$ (using quadratics to be discussed in Secs. V and IV), the $E_{0}$ parameter of Eq. (6) lowers the HSE06 gap by $258 \mathrm{meV}$ (accounting for DFT inaccuracies and perturbative phenomena), while suggesting average phonon and interaction energies $\left\{E_{i}, \alpha_{i}\right\}$ of $\{36.95,168.69\}$ and $\{19.34,-77.75\} \mathrm{meV}$ (see Table I), which are perhaps somewhat improved over the plain dual BoseEinstein oscillator, see again Fig. 3(b).

Each of the Bose-Einstein oscillator models suggests some counterplay between the parameter $E_{0}$ and the signs of the electron-phonon interaction $\alpha_{i}$ terms, which gives some freedom to fit the experimental data. Equation (16) in the appendix of the Supplemental Material [49] permits different signs for the $\alpha_{\mathrm{ep}}$ terms, particularly for phonon energies of different magnitudes.

\section{STRUCTURAL STUDIES}

Reflections associated with the well-known cubic $P m 3 m$ anti- $\mathrm{ReO}_{3}$ phase appear in all diffraction patterns, with little 

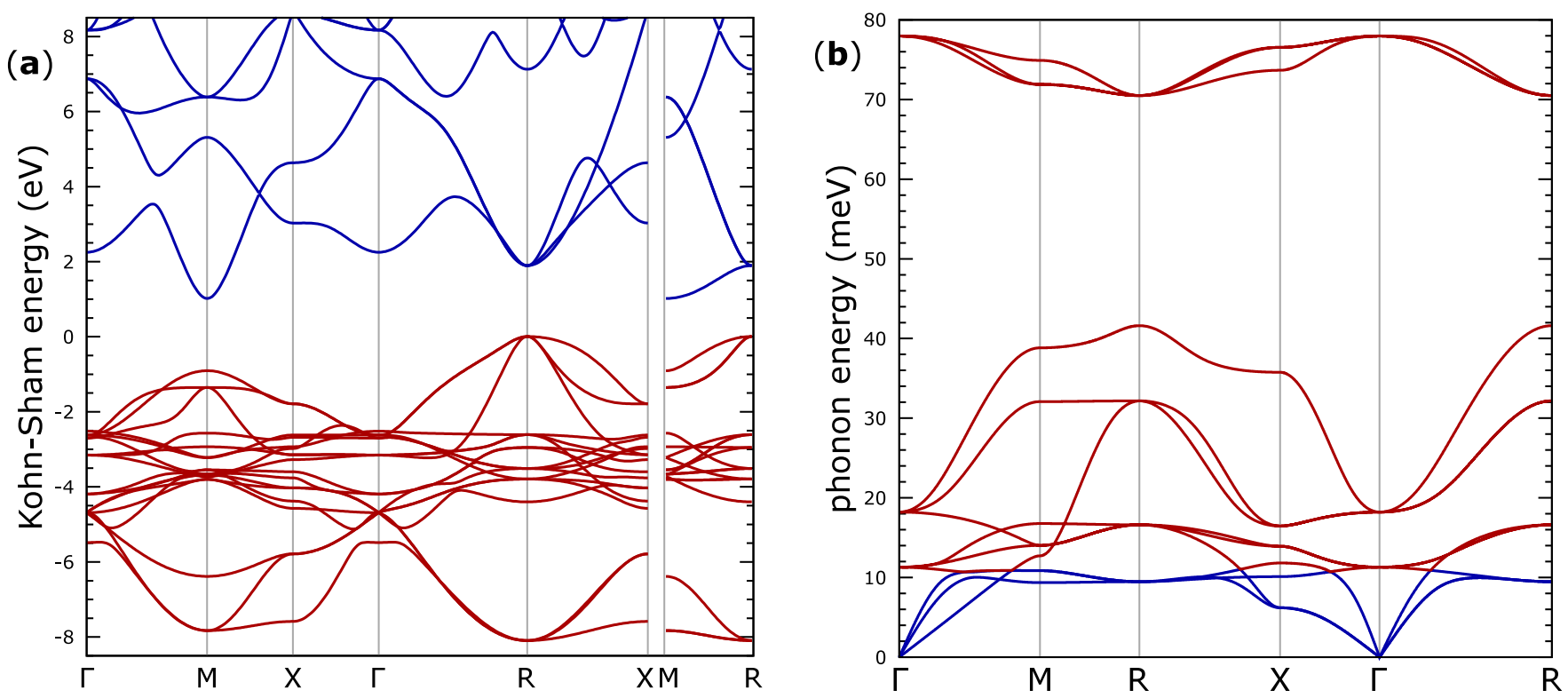

FIG. 3. Band structure and phonon dispersion. (a) Valence-band maximum and conduction-band minimum are respectively at the $R$ and $M$ points in the HSE06 band structure; the smallest direct gap lies between $R$ and $M$. (b) Depicts the PBEsol phonon dispersion; the density of states has significant maxima at 10 and $75 \mathrm{meV}$. The IR-active $\Gamma_{15}$ phonon state at $78.0 \mathrm{meV}$ is associated with a feature in the experimental FTIR spectra, see the inset of Fig. 3 in the Supplemental Material [49].

temperature dependence between 4.2 and $280 \mathrm{~K}$. Weak reflections from the beryllium cryostat domes are apparent in the $\mathrm{Cu} K \alpha$ pattern at $4.2 \mathrm{~K}$ in Fig. 4(a); these reflections are shifted from their Bragg positions because the domes are displaced from the diffractometer axis [61]. Refinement of all patterns suggested $(m 00)$ preferential orientation, evident in the mismatched Mo $K \alpha$ pattern (200) residuals in Fig. 4(a). Preferential orientation in the Mo $K \alpha$ patterns likely results from incomplete separation of the $\mathrm{Cu}_{3} \mathrm{~N}$ crystallites after removal from the substrate. Considering that proceeding with a Rietveld approach without an acceptable structural model would drive errors elsewhere in the fit, particularly in lattice parameters and their uncertainties, a Pawley-like [62,63] full-pattern fit was instead implemented in MATLAB with the reflection intensities fit as free parameters.

The thermal expansion determined for $\mathrm{Cu}_{3} \mathrm{~N}$ is plotted in Fig. 4(b). The lattice parameter as measured contracts respectively from $3.82305(24)$ to $3.8181(3) \AA$ between 280 and $100 \mathrm{~K}$; below $100 \mathrm{~K}$ the magnitude of thermal expansion reduces significantly, with a $3.8173(6) \AA$ parameter seen at $4.2 \mathrm{~K}$. Indeed, the thermal expansion below $150 \mathrm{~K}$ is sufficiently small that the 4.2 and $150 \mathrm{~K}$ lattice parameters are consistent (within $3 \sigma$ ). The consistency in the $100 \mathrm{~K}$ lattice parameter from instruments in different laboratories lends some confidence to the results. Despite finding negligible expansion below $100 \mathrm{~K}$, the results are not sufficiently precise to confirm or reject the possibility of zero or negative thermal expansion here.

To evaluate the thermal expansion, the measured lattice parameters are fit to a quasiharmonic Debye-EinsteinGrüneisen model (see the Supplemental Material [49]). The lattice expansion and thermal (linear) expansion coefficient are plotted in Fig. 4(b). Alternatively, fitting the expansion to a quadratic in $T(\mathrm{~K})$ suggests (with uncertainties indicated in brackets)

$$
\begin{aligned}
a(\AA)= & 3.81807(22)-1.1(3) \times 10^{-5} T \\
& +1.01(11) \times 10^{-7} T^{2} .
\end{aligned}
$$

The fit also finds a Debye temperature of 550(150) K, a zero-kelvin lattice parameter of 3.817 88(19) $\AA$ and an average Grüneisen parameter of 2.3(9). Inserting the fit $\alpha_{\mathrm{V}}$ and the unitcell volume back into Supplemental Material Eq. (1) [49] and solving for $C_{\mathrm{V}}$ gives respective lattice isochoric heat capacities of 43 and $300 \mathrm{~J} \mathrm{~kg}^{-1} \mathrm{~K}^{-1}$ at 100 and $300 \mathrm{~K}$; see the inset in Fig. 4(b). Standard thermodynamic relations then estimate an isobaric heat capacity $C_{\mathrm{P}}$ of $325 \mathrm{~J} \mathrm{~kg}^{-1} \mathrm{~K}^{-1}$ at $300 \mathrm{~K}$.

\section{THEORETICAL STUDIES}

Much of the previous DFT work on $\mathrm{Cu}_{3} \mathrm{~N}$ uses functionals likely to inaccurately determine the band gap [64-66]. Evaluation of the HSE06 band structure along a reciprocal space path tracing all edges of the irreducible primitive cubic Brillouin zone [67] [see Fig. 3(a)] shows a band dispersion and density of states rather consistent with those seen elsewhere [13]. The valence-band maximum (VBM) and conduction-band minimum (CBM) are found respectively at the $R\left(\frac{1}{2} \frac{1}{2} \frac{1}{2}\right)$ and $M\left(\frac{1}{2} \frac{1}{2} 0\right)$ symmetry points. The direct and indirect gaps are 1.726 and $1.014 \mathrm{eV}$ at the relaxed volume (lattice parameter $3.827 \AA$ ), with the smallest direct gap lying between $R$ and $M$. This VBM and CBM configuration is widely reported for $\mathrm{Cu}_{3} \mathrm{~N}$; however, because important symmetry lines are often omitted, particularly the important line between $R$ and $M$, the smallest direct gap may occasionally be claimed erroneously at $R$. The lowest direct gap between $R$ and $M$ in Fig. 3(a) is not seen in the GW band structure of Ref. [13] due to slight differences in the curvature of the lowest conduction band. 

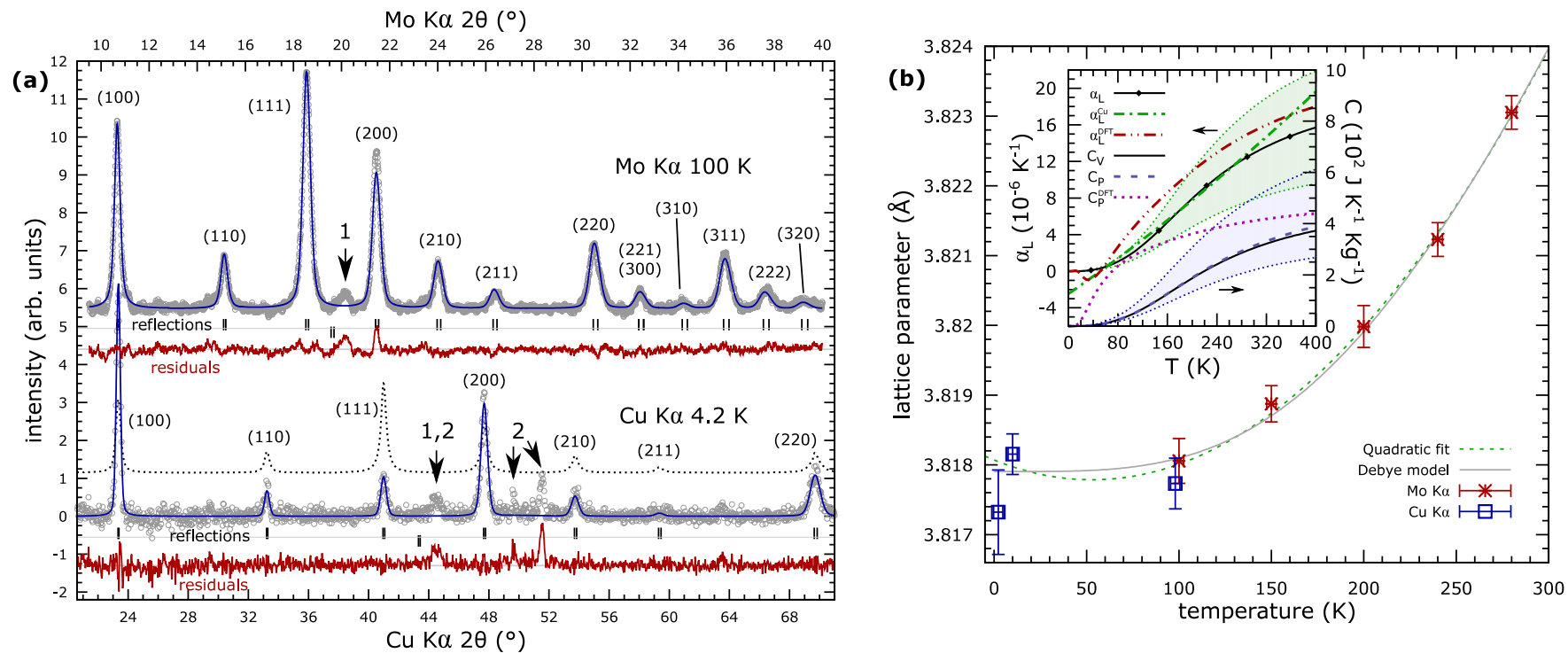

FIG. 4. Temperature-dependent x-ray diffraction and thermal expansion of $\mathrm{Cu}_{3} \mathrm{~N}$. (a) Full-pattern fits to background-subtracted Mo $K \alpha$ and $\mathrm{Cu} K \alpha$ patterns respectively at 100 and $4.2 \mathrm{~K}$. Displaced reflections from the beryllium cryostat windows (labeled 2$)$ are seen at $44^{\circ}$ through $52^{\circ} 2 \theta$ at $4.2 \mathrm{~K}$; an unidentified feature near $20^{\circ} 2 \theta$ Mo $K \alpha$ or $44.5^{\circ} 2 \theta \mathrm{Cu} K \alpha$ (labeled 1) is discussed in the Supplemental Material [49]. Rietveld refinement fits the Mo $K \alpha$ pattern acceptably at $100 \mathrm{~K}$, but gives a poor fit (dotted line, displaced vertically for clarity) to the $\mathrm{Cu}$ $K \alpha 4.2 \mathrm{~K}$ pattern due to preferential $(\mathrm{m} 00$ ) orientation. Pawley refinement fits the $4.2 \mathrm{~K}$ pattern successfully after excluding the beryllium lines. (b) Plots of cubic lattice parameters fit by Pawley refinement, with the lattice parameter evolution fit by a quadratic and a quasiharmonic Grüneisen-Debye-Einstein model. The inset compares the quasiharmonic thermal linear expansion $\alpha_{L}$ (and a cubic fit $\alpha_{L}^{\mathrm{Cu}}$ ) and heat capacities $C_{\mathrm{V}}$ and $C_{\mathrm{P}}$ with those from first-principles calculations $\left(\alpha_{\mathrm{L}}^{\mathrm{DFT}}\right.$ and $\left.C_{\mathrm{P}}^{\mathrm{DFT}}\right)$. The filled regions around $\alpha_{\mathrm{L}}$ and $C_{\mathrm{V}}$ denote the $1 \sigma$ uncertainties, which are dominated by the Grüneisen parameter uncertainty. Negligible thermal expansion is seen below $100 \mathrm{~K}$.

A parabolic fit finds an isotropic hole effective mass of $0.19 m_{\mathrm{e}}$ at $R$, while strong dispersion is seen in the CBM with significant anisotropy in the electron effective masses: with a light average of $0.16 m_{\mathrm{e}}$ in the $M-\Gamma$ and $M-X$ directions, but a significantly heavier $1.60 m_{\mathrm{e}}$ in the $M-R$ direction. The electron and hole effective masses are notably low (lower than the density of states effective masses fit in Ref. [13]) and indicate potentially high carrier mobilities, beneficial for photovoltaic carrier collection (particularly noting the bipolar capability of $\mathrm{Cu}_{3} \mathrm{~N}$ ). The dependence of the direct band gap (in $\mathrm{eV}$ ) on lattice parameter (in $\AA$, between 3.8 and $3.89 \AA$ ) was fit to a quadratic $a x^{2}+b x+c$ with $\{a, b, c\}=$ $\{-1.38,13.24,-28.57\}$.

Optical absorption and refractive index spectra derived from HSE06 are shown in Fig. 1; these spectra are shifted by $0.5 \mathrm{eV}$ to lower photon energies to reveal remarkable similarities with the experimental ellipsometric spectra (factors which may introduce such a shift are discussed in the next section). Some small, fictitious subgap absorption results from a phase shift which avoids a singularity in the evaluation of the Kramers-Kronig transform. Although the smallest direct gap lies between $R$ and $M$, the electric-dipole matrix element vanishes along this line indicating that such transitions here are symmetry forbidden. The absorption onset is thus associated with a $1.890 \mathrm{eV}$ transition at $R$. While the HSE06 band structure is strongly supported by the shifted spectra of Fig. 1, the uncorrected spectra suggest an optical absorption coefficient at $2 \mathrm{eV}$ which is just a quarter of that found experimentally (both here and elsewhere [10]). In first-principles searches for new photovoltaic materials, this perhaps indicates the need to estimate the impact of omitted phenomena that may contribute significantly.

The phonon dispersion curve in Fig. 3(b) was evaluated using the finite-difference approach with PBEsol. At the zone center, in addition to the acoustic modes (blue), there are three triply degenerate optical phonon bands (red), two of which are predicted to be IR active with phonon energies of 11.3 and $78.0 \mathrm{meV}$, with density of states maxima near 10 and $75 \mathrm{meV}$. The lack of imaginary or negative frequency modes indicates the stability of the $\mathrm{Cu}_{3} \mathrm{~N}$ structure. This phonon dispersion is rather similar to that reported by $\mathrm{Yu}$ et al. [59], with lower phonon energies likely due to the lower effect of underbinding found in PBEsol in comparison to LDA.

The quasiharmonic approximation (QHA) was used to estimate thermal effects in $\mathrm{Cu}_{3} \mathrm{~N}$ below $500 \mathrm{~K}$. This involved phonon dispersion calculations for various cell volumes, giving expansions and contractions of the equilibrium cell volume. From this, energy-volume curves corresponding to a range of temperatures are constructed, and the volumes fit to the Birch-Murnaghan equation of state [68]. The resulting linear thermal expansion and isobaric heat capacity are seen in the inset of Fig. 4(b). Below $50 \mathrm{~K}$, negative thermal expansion is predicted, but this is not supported or ruled out by the experimental results; at higher temperatures, the DFT expansion coefficient matches well to the experimental Debye model. This contrasts with the $\mathrm{ReO}_{3}$-structured $\mathrm{ScF}_{3}$, where strong anharmonicity leads to large variations in predicted and observed thermal expansion [69,70]. In other semiconductors, 
TABLE I. Temperature-dependence of the direct gap of $\mathrm{Cu}_{3} \mathrm{~N}$, described by various models. See Eqs. (5)-(7). All units are meV except the Varshni $\alpha$ and $\beta$ parameters, which have units $\mathrm{meV} / \mathrm{K}$ and $\mathrm{K}$, respectively.

\begin{tabular}{lcc}
\hline \hline Model & Parameter & Value \\
\hline Varshni & $E_{0}$ & 1700 \\
& $\alpha$ & 0.13 \\
& $\beta$ & 0.014 \\
Bose-Einstein & $E_{0}$ & $1540(130)$ \\
(two oscillators) & $E_{\mathrm{ph}, 1}$ & $10(6)$ \\
& $\alpha_{1}$ & $-17(40)$ \\
& $E_{\mathrm{ph}, 2}$ & $52(24)$ \\
& $\alpha_{2}$ & $180(100)$ \\
Bose-Einstein & $E_{\mathrm{g}}^{\mathrm{direct}}(a)$ & See Table II \\
(plus lattice & $a(T)$ & See Table II \\
expansion) & $E_{0}$ & -258 \\
& $E_{\mathrm{ph}, 1}$ & 36.95 \\
& $\alpha_{1}$ & 168.69 \\
& $E_{\mathrm{ph}, 2}$ & 19.34 \\
& $\alpha_{2}$ & -77.75 \\
\hline \hline
\end{tabular}

overestimated DFT- and QHA-predicted thermal expansion has been attributed to higher-order anharmonic effects [71]. The experimental and predicted $\mathrm{Cu}_{3} \mathrm{~N}$ heat capacities also differ significantly below $140 \mathrm{~K}$; the opposite curvatures likely result from the predicted negative thermal expansion: at higher temperatures, the predicted $C_{\mathrm{P}}$ tends towards the Debye fit.

\section{DISCUSSION}

The experimental and shifted HSE06 spectra of Fig. 1 display convincingly similar refractive index and absorption features: both in terms of gradients and in absolute and relative feature positions. In previous reports of rigid shifts between experimental and DFT absorption spectra in indium nitride [72], displacements were explained and corrected through inclusion of the electron-hole interaction, which is omitted in the calculations of Fig. 1. In InN, excitonic interactions lower the computed spectra by $\sim 0.5 \mathrm{eV}$ while introducing minor features at lower photon energies. Such excitonic effects perhaps account substantially for the magnitude of the shift seen in Fig. 1. Additional contributing factors may include the $0.2 \mathrm{eV}$ difference between the experimental and HSE06-predicted direct band gaps, and the omission of indirect transitions (which would lower each onset). Minor differences in the spectra, such as the different curvatures in the refractive index spectra at $2.6 \mathrm{eV}$, may be due to either excitonic effects or phonon-assisted transitions.

Despite the similarities of the spectra in Fig. 1, the finding of dipole-forbidden transitions along $R-M$ may be more speculative. It is notable that the $1.68 \mathrm{eV}$ direct gap found experimentally by FTIR and ellipsometry is rather closer to the HSE06 $1.726 \mathrm{eV}$ direct gap between $R$ and $M$. Further work is needed to address this question. In any case, the photovoltaic performance of $\mathrm{Cu}_{3} \mathrm{~N}$ is not necessarily entirely compromised by a $1.0 \mathrm{eV}$ indirect gap (considering the $1.1 \mathrm{eV}$ gap of silicon) or the presence of dipole-forbidden transitions along $R-M$, particularly as the dipole-allowed transition at $R$ is just $\sim 0.16 \mathrm{eV}$ to higher energy. Once inside the conduction band at $R$, electrons relax [73] by $\sim 0.8 \mathrm{eV}$ into the CBM at $M$, where radiative recombination is limited by weak indirect transitions (too few holes are available at $M$ to activate the $1.925 \mathrm{eV}$ direct transition here). While the absorption edge of $\mathrm{Si}$ increases weakly until the direct onset at $3.2 \mathrm{eV}$, the absorption in $\mathrm{Cu}_{3} \mathrm{~N}$ is significantly boosted by the direct transition at $\sim 1.8 \mathrm{eV}$, supporting potential applications for $\mathrm{Cu}_{3} \mathrm{~N}$ in thin-film photovoltaics.

The atypically small $24 \mathrm{meV}$ redshift of the direct gap between 4.2 and $300 \mathrm{~K}$ may be related to the small HSE06 band-edge effective masses; the negative conduction-band effective mass at the direct onset at $R$ perhaps also contributes. Bands can be perturbed in different directions (as a function of temperature) by the Fan and Debye-Waller terms of the electron-phonon interaction (supporting the signs of the interaction energies found earlier), which may ultimately lead to any shifts canceling out $[56,74,75]$. While a small redshift is clear in the absorption of Fig. 2(a), the functional form of the temperature-evolution is somewhat atypical. While anomalies might arise from fitting the featureless absorption edge of Fig. 2(a), atypical band-gap temperature-dependence has been previously explained as due to lattice expansion [55] or (in perovskites) to distinctly evolving direct and indirect gaps [76]. In $\mathrm{AgGaSe}_{2}$ a similarly small $30 \mathrm{meV}$ redshift of an unambiguous direct gap and anomalous temperature evolution was found between 15 and $300 \mathrm{~K}$ and explained as arising from negative thermal expansion (also predicted here for $\mathrm{Cu}_{3} \mathrm{~N}$ ) [77]. More generally, anomalous temperaturedependence seen in silver and copper containing chalcopyrites, e.g., $\mathrm{AgGaSe}_{2}$ or $\mathrm{CuGaS}_{2}$, is hypothesized as related to valence-band $p$ - $d$ hybridization [55], and characterized by maxima in $E_{\mathrm{g}}(T)$ well above $0 \mathrm{~K}$ (fit similarly with two BoseEinstein oscillators). Prior band character studies in $\mathrm{Cu}_{3} \mathrm{~N}$ find strong $p-d$ and $s$ - $d$ hybridization in the respective valence and conduction bands, perhaps supporting the hypothesized culpability of the $\mathrm{Cu}-3 d$ valence electrons [13].

The lattice parameters determined in this work below $280 \mathrm{~K}$ are consistent with those of Zachwieja et al. [29], who studied single crystals, but are inconsistent with those for the microcrystalline material of Wosylus et al. [28] and for the powders of Juza and Hahn [14]. The thermal linear lattice expansion determined by a quasiharmonic Debye-Einstein-Grüneisen model decreases monotonically from $1.3 \times 10^{-5} \mathrm{~K}^{-1}$ at $300 \mathrm{~K}$; below $100 \mathrm{~K}$ the expansion diminishes significantly and falls below $1.8 \times 10^{-6} \mathrm{~K}^{-1}$. This is consistent with the $0.005 \AA$ reported in the EXAFS study of Kuzmin et al. [31] between 10 and $300 \mathrm{~K}$; however, a suggested $6.4(3) \times 10^{-6} \mathrm{~K}^{-1}$ linear thermal expansion coefficient [28] perhaps misrepresents the thermal expansion in $\mathrm{Cu}_{3} \mathrm{~N}$ and should possibly be viewed as an average expansion across the range. Our thermal expansion results are of similar order to those seen in the structurally similar anti- $\mathrm{ReO}_{3}$ compound $\mathrm{Na}_{3} \mathrm{~N}$, for which powder and single-crystal samples have been studied by x-ray and neutron diffraction [78], and a linear expansion coefficient of $4.6 \times 10^{-6} \mathrm{~K}^{-1}$ is found between 20 and $293 \mathrm{~K}$. 
TABLE II. Summary of various quantities found in this work

\begin{tabular}{|c|c|}
\hline Quantity & Value \\
\hline \multicolumn{2}{|c|}{$\begin{array}{l}\text { Lattice parameter (in } \AA \text { ) temperature dependence (in K), } \\
1.01(11) \times 10^{-7} T^{2}-1.1(3) \times 10^{-5} T+3.81807(22)\end{array}$} \\
\hline \multicolumn{2}{|c|}{ Quasiharmonic parameters fitted to XRD: } \\
\hline Debye temperature & $550(150) \mathrm{K}$ \\
\hline Einstein temperature & $825(225) \mathrm{K}$ \\
\hline $0 \mathrm{~K}$ lattice parameter & $3.81788(19) \AA$ \\
\hline Grüneisen parameter & $2.3(9)$ \\
\hline Bulk modulus & $110 \mathrm{GPa}$ \\
\hline \multicolumn{2}{|l|}{ Heat capacities } \\
\hline$C_{V}$ at $100 \mathrm{~K}$ & $43 \mathrm{~J} \mathrm{~kg}^{-1} \mathrm{~K}^{-1}$ \\
\hline$C_{V}$ at $300 \mathrm{~K}$ & $300 \mathrm{~J} \mathrm{~kg}^{-1} \mathrm{~K}^{-1}$ \\
\hline$C_{p}$ at $300 \mathrm{~K}$ & $325 \mathrm{~J} \mathrm{~kg}^{-1} \mathrm{~K}^{-1}$ \\
\hline \multicolumn{2}{|l|}{ HSE06 band structure } \\
\hline$E_{\mathrm{g}}^{\text {direct }}$ & $1.726 \mathrm{eV}$ along $R-M$ \\
\hline$E_{\mathrm{g}}^{\mathrm{direct}}(a$ in $\AA)$ in $\mathrm{eV}$ & $-1.38 a^{2}+13.24 a-28.57$ \\
\hline$E_{\mathrm{g}}^{\mathrm{optical}}$ & $1.890 \mathrm{eV}$ at $R$ \\
\hline$E_{\mathrm{g}}^{\text {indirect }}$ & $1.014 \mathrm{eV}$ from $R$ to $M$ \\
\hline$m_{v}^{*}(\mathrm{R})$ & $0.19 m_{e}$ \\
\hline$m_{c}^{*}(M-\Gamma), m_{c}^{*}(M-X)$ & $0.16 m_{e}$ \\
\hline$m_{c}^{*}(M-R)$ & $1.60 m_{e}$ \\
\hline
\end{tabular}

Lattice heat capacities $C_{\mathrm{V}}$ of 43 and $300 \mathrm{~J} \mathrm{~kg}^{-1} \mathrm{~K}^{-1}$ were determined at 100 and $300 \mathrm{~K}$ (see also Table II). The heat capacity at $300 \mathrm{~K}$ is of reasonable magnitude considering again the related compound $\mathrm{Na}_{3} \mathrm{~N}$, for which a $720 \mathrm{~J} \mathrm{~kg}^{-1} \mathrm{~K}^{-1}$ heat capacity $C_{\mathrm{V}}$ is predicted by a Debye model [79]. Heat capacities tend to decrease with increasing atomic mass [80,81]: those for $\mathrm{Si}, \mathrm{Ge}, \mathrm{Sn}, \mathrm{Pb}, \mathrm{GaP}, \mathrm{GaAs}$, and $\mathrm{GaSb}$ respectively evolve as 705, 320, 228, 129, 440, 320, and $254 \mathrm{~J} \mathrm{~kg}^{-1} \mathrm{~K}^{-1}$. Above $150 \mathrm{~K}$, the experimental lattice expansion and heat capacity are rather consistent with those determined by the quasiharmonic approximation. Below $50 \mathrm{~K}$ these calculations suggest negative thermal expansion [82], which is feasible in perovskite-like structures due to rigid-unit modes [83] (particularly at low temperature), but this is not supported or ruled out by the present (or prior) work, which finds no structural phase transitions from the cubic $P m 3 m$ structure.

\section{CONCLUSION}

Optical and structural investigations reveal atypically small temperature evolution of the direct gap in copper nitride between 4.2 and $300 \mathrm{~K}$. Fourier-transform infrared spectroscopy and spectroscopic ellipsometry determine a strong absorption onset associated with a $1.68 \mathrm{eV}$ direct gap at $300 \mathrm{~K}$, preceded by contributions from a weak indirect onset and Urbach tail. The absorption strength reaches a magnitude of $3.5 \times 10^{5} \mathrm{~cm}^{-1}$ at $2.7 \mathrm{eV}$, suggesting potential for photovoltaic applications. The redshift of the direct gap is just $24 \mathrm{meV}$ between 4.2 and $300 \mathrm{~K}$, rather less than seen typically in semiconductors. The temperature-dependence of the direct gap is described by various models (see Table I), with quite reasonable average phonon energies upon consideration of maxima in the phonon density of states. Future work should seek to determine the lattice expansion with improved accuracy. Very little expansion is seen in $\mathrm{Cu}_{3} \mathrm{~N}$ below $80 \mathrm{~K}$; the use of either single crystals or high-precision instruments (i.e., measuring to high $2 \theta$ ) with good signal to noise, e.g., two-dimensional detectors or synchrotron sources, seems advisable for further work.

\section{ACKNOWLEDGMENTS}

This work was supported by UK Engineering and Physical Sciences Research Council (EPSRC) Grants EP/K503095/1 and EP/J50047/1, and by EPSRC funding of the offline $\mathrm{X}$-ray facility of the XMaS beamline at the European Synchrotron Radiation Facility. The computational work was performed on the UCL Legion HPC Facility (Legion@UCL), UCL Grace HPC Facility (Grace@UCL), and the Archer UK National Supercomputing Service via membership of the UK's HEC Materials Chemistry Consortium, which is funded by EPSRC (EP/L000202). A.N.F. and A.Z. acknowledge funding from the U.S. Department of Energy under Contract No. DE-AC36-08GO28308 to NREL. C.N.S. acknowledges the Department of Chemistry at UCL for the provision of a DTA studentship. T.D.V. and D.O.S. acknowledge membership of the Materials Design Network. Mohana Rajpalke at the Norwegian University of Science and Technology (formally at Liverpool) is thanked for assistance with SEM measurements.
[1] M. Asano, K. Umeda, and A. Tasaki, Jpn. J. Appl. Phys. 29, 1985 (1990).

[2] T. Maruyama and T. Morishita, Appl. Phys. Lett. 69, 890 (1996).

[3] R. Cremer, M. Witthaut, D. Neuschütz, C. Trappe, M. Laurenzis, O. Winkler, and H. KurZ, Microchim. Acta 133, 299 (2000).

[4] Q. Lu, X. Zhang, W. Zhu, Y. Zhou, Q. Zhou, L. Liu, and X. Wu, Phys. Status Solidi A 208, 874 (2011).

[5] C. Navío, M. J. Capitán, J. Álvarez, F. Yndurain, and R. Miranda, Phys. Rev. B 76, 085105 (2007).

[6] D. M. Borsa, S. Grachev, C. Presura, and D. O. Boerma, Appl. Phys. Lett. 80, 1823 (2002).

[7] L. Maya, J. Vac. Sci. Technol., A 11, 604 (1993).

[8] L. Maya, MRS Online Proc. Libr. 282, 203 (1992).
[9] T. Nosaka, M. Yoshitake, A. Okamoto, S. Ogawa, and Y. Nakayama, Appl. Surf. Sci. 169-170, 358 (2001).

[10] C. M. Caskey, R. M. Richards, D. S. Ginley, and A. Zakutayev, Mater. Horiz. 1, 424 (2014).

[11] K. Matsuzaki, T. Okazaki, Y.-S. Lee, H. Hosono, and T. Susaki, Appl. Phys. Lett. 105, 222102 (2014).

[12] A. N. Fioretti, C. P. Schwartz, J. Vinson, D. Nordlund, D. Prendergast, A. C. Tamboli, C. M. Caskey, F. Tuomisto, F. Linez, S. T. Christensen, E. S. Toberer, S. Lany, and A. Zakutayev, J. Appl. Phys. 119, 181508 (2016).

[13] A. Zakutayev, C. M. Caskey, A. N. Fioretti, D. S. Ginley, J. Vidal, V. Stevanovic, E. Tea, and S. Lany, J. Phys. Chem. Lett. 5, 1117 (2014). 
[14] R. Juza and H. Hahn, Z. Anorg. Allg. Chem. 239, 282 (1938).

[15] R. Gonzalez-Arrabal, N. Gordillo, M. S. Martin-Gonzalez, R. Ruiz-Bustos, and F. Agulló-López, J. Appl. Phys. 107, 103513 (2010).

[16] Y. Du, A. L. Ji, L. B. Ma, Y. Q. Wang, and Z. X. Cao, J. Cryst. Growth 280, 490 (2005).

[17] D. Dorranian, L. Dejam, A. H. Sari, and A. Hojabri, Eur. Phys. J.: Appl. Phys. 50, 20503 (2010).

[18] I. M. Odeh, J. Alloys Compd. 454, 102 (2008).

[19] N. Gordillo, R. Gonzalez-Arrabal, A. Álvarez-Herrero, and F. Agulló-López, J. Phys. D: Appl. Phys. 42, 165101 (2009).

[20] T. Maruyama and T. Morishita, J. Appl. Phys. 78, 4104 (1995).

[21] F. Hadian, A. Rahmati, H. Movla, and M. Khaksar, Vacuum 86, 1067 (2012).

[22] A. Fallberg, M. Ottosson, and J.-O. Carlsson, Chem. Vap. Deposition 15, 300 (2009).

[23] K. J. Kim, J. H. Kim, and J. H. Kang, J. Cryst. Growth 222, 767 (2001).

[24] Y. Du, R. Huang, R. Song, L. B. Ma, C. Liu, C. R. Li, and Z. X. Cao, J. Mater. Res. 22, 3052 (2007).

[25] A. Zakutayev, J. Mater. Chem. A 4, 6742 (2016).

[26] P. Singh and N. Ravindra, Sol. Energy Mater. Sol. Cells 101, 36 (2012).

[27] P. Löper, D. Pysch, A. Richter, M. Hermle, S. Janz, M. Zacharias, and S. Glunz, Energy Procedia 27, 135 (2012).

[28] A. Wosylus, U. Schwarz, L. Akselrud, M. G. Tucker, M. Hanfland, K. Rabia, C. Kuntscher, J. von Appen, R. Dronskowski, D. Rau, and R. Niewa, Z. Anorg. Allg. Chem. 635, 1959 (2009).

[29] U. Zachwieja and H. Jacobs, J. Less-Common Met. 161, 175 (1990)

[30] G. Paniconi, Z. Stoeva, H. Doberstein, R. I. Smith, B. L. Gallagher, and D. H. Gregory, Solid State Sci. 9, 907 (2007).

[31] A. Kuzmin, A. Kalinko, A. Anspoks, J. Timoshenko, and R. Kalendarev, EXAFS Study of Local Structure and Lattice Dynamics in Copper Nitride $\mathrm{Cu}_{3} \mathrm{~N}$, Tech. Rep. (Institute of Solid State Physics, University of Latvia, 2012).

[32] Corning Inc., Corning Eagle XG Product Information Sheet, Tech. Rep. (Corning Display Technologies, 2013).

[33] R. D. Deslattes, E. G. Kessler Jr., P. Indelicato, and E. Lindroth, in International Tables for Crystallography Volume C: Mathematical, Physical and Chemical Tables, 2006 ed. (International Union of Crystallography, Dordrecht, 2006), Chap. 4.2.2, pp. 200-212.

[34] G. Kresse and J. Furthmüller, Phys. Rev. B 54, 11169 (1996).

[35] G. Kresse and J. Hafner, Phys. Rev. B 49, 14251 (1994).

[36] G. Kresse and J. Hafner, Phys. Rev. B 47, 558 (1993).

[37] G. Kresse and J. Furthmüller, Comput. Mater. Sci. 6, 15 (1996).

[38] J. Heyd, G. E. Scuseria, and M. Ernzerhof, J. Chem. Phys. 118, 8207 (2003).

[39] J. E. Peralta, J. Heyd, G. E. Scuseria, and R. L. Martin, Phys. Rev. B 74, 073101 (2006).

[40] A. V. Krukau, O. A. Vydrov, A. F. Izmaylov, and G. E. Scuseria, J. Chem. Phys. 125, 224106 (2006).

[41] P. E. Blöchl, Phys. Rev. B 50, 17953 (1994).

[42] G. Kresse and D. Joubert, Phys. Rev. B 59, 1758 (1999).

[43] A. Togo, F. Oba, and I. Tanaka, Phys. Rev. B 78, 134106 (2008).

[44] A. Togo and I. Tanaka, Scr. Mater. 108, 1 (2015).
[45] G. I. Csonka, J. P. Perdew, A. Ruzsinszky, P. H. T. Philipsen, S Lebègue, J. Paier, O. A. Vydrov, and J. G. Ángyán, Phys. Rev. B 79, 155107 (2009).

[46] J. P. Perdew, A. Ruzsinszky, G. I. Csonka, O. A. Vydrov, G. E. Scuseria, L. A. Constantin, X. Zhou, and K. Burke, Phys. Rev. Lett. 100, 136406 (2008).

[47] E. L. da Silva, J. M. Skelton, S. C. Parker, and A. Walsh, Phys. Rev. B 91, 144107 (2015).

[48] M. Gajdoš, K. Hummer, G. Kresse, J. Furthmüller, and F. Bechstedt, Phys. Rev. B 73, 045112 (2006).

[49] See Supplemental Material at http://link.aps.org/supplemental/ 10.1103/PhysRevB.95.115201 for more details on the optical and XRD fitting.

[50] P. Lautenschlager, M. Garriga, S. Logothetidis, and M. Cardona, Phys. Rev. B 35, 9174 (1987).

[51] P. Y. Yu and M. Cardona, Fundamentals of Semiconductors, 4th ed. (Springer, Berlin, Heidelberg, 2010).

[52] K. P. O'Donnell and X. Chen, Appl. Phys. Lett. 58, 2924 (1991).

[53] P. M. Amirtharaj and D. G. Seiler, Handbook of Optics, 2nd ed (McGraw-Hill, New York, 1995), Vol. 2, Chap. 36, Table 13.

[54] Y. P. Varshni, Physica 34, 149 (1967).

[55] J. Bhosale, A. K. Ramdas, A. Burger, A. Muñoz, A. H. Romero, M. Cardona, R. Lauck, and R. K. Kremer, Phys. Rev. B 86, 195208 (2012).

[56] C. Yu, Z. Chen, J. J. Wang, W. Pfenninger, N. Vockic, J. T. Kenney, and K. Shum, J. Appl. Phys. 110, 063526 (2011).

[57] A. Göbel, T. Ruf, M. Cardona, C. T. Lin, J. Wrzesinski, M. Steube, K. Reimann, J.-C. Merle, and M. Joucla, Phys. Rev. B 57, 15183 (1998).

[58] S. Adachi, Properties of Group-IV, III-V and II-VI Semiconductors, 1st ed. (Wiley, Chichester, 2005).

[59] W. Yu, J. Zhao, and C. Jin, Phys. Rev. B 72, 214116 (2005).

[60] M. Cardona and R. Kremer, Thin Solid Films 571, 680 (2014).

[61] The strongest beryllium reflections (100), (002), and (101) are expected $[84,85]$ respectively at $45.8^{\circ}, 50.9^{\circ}$, and $52.8^{\circ} 2 \theta$, while the observed reflections lie at $44.5^{\circ}, 49.6^{\circ}$, and $51.5^{\circ}$ $(\mathrm{Cu} K \alpha)$. These angles do not change [86] significantly below $300 \mathrm{~K}$. The expected and observed angles are then consistent within $0.04^{\circ} 2 \theta$ via the relation [87] for sample displacement, $\Delta 2 \theta(\mathrm{rad})=(2 s / R) \cos (1 \theta)$, with the ratio $2 s / R=-0.0255$.

[62] G. S. Pawley, J. Appl. Crystallogr. 13, 630 (1980).

[63] G. S. Pawley, J. Appl. Crystallogr. 14, 357 (1981).

[64] H. Chen, X. Li, J. Zhao, Z. Wu, T. Yang, Y. Ma, W. Huang, and K. Yao, Comput. Theor. Chem. 1018, 71 (2013).

[65] F. Kong, Y. Hu, Y. Wang, B. Wang, and L. Tang, Comput. Mater. Sci. 65, 247 (2012).

[66] M. G. Moreno-Armenta, A. Martínez-Ruiz, and N. Takeuchi, Solid State Sci. 6, 9 (2004).

[67] W. Setyawan and S. Curtarolo, Comput. Mater. Sci. 49, 299 (2010).

[68] F. Birch, Phys. Rev. 71, 809 (1947).

[69] C. W. Li, X. Tang, J. A. Muñoz, J. B. Keith, S. J. Tracy, D. L. Abernathy, and B. Fultz, Phys. Rev. Lett. 107, 195504 (2011).

[70] L. Hu, J. Chen, A. Sanson, H. Wu, C. G. Rodriguez, L. Olivi, Y. Ren, L. Fan, J. Deng, and X. Xing, J. Am. Chem. Soc. 138, 8320 (2016). 
[71] J. M. Skelton, S. C. Parker, A. Togo, I. Tanaka, and A. Walsh, Phys. Rev. B 89, 205203 (2014).

[72] J. Furthmüller, F. Fuchs, and F. Bechstedt, in Indium Nitride and Related Alloys, 1st ed., edited by T. D. Veal, C. F. McConville, and W. J. Schaff (CRC Press, Boca Raton, 2010), Chap. 8.4.

[73] A. M. Fox, Quantum Optics: An Introduction, 1st ed. (Oxford University Press, Oxford, 2006), Chap. 4.6.

[74] P. B. Allen and V. Heine, J. Phys. C: Solid State Phys. 9, 2305 (1976).

[75] M. Cardona, Solid State Commun. 133, 3 (2005).

[76] M. C. Weber, M. Guennou, C. Toulouse, M. Cazayous, Y. Gillet, X. Gonze, and J. Kreisel, Phys. Rev. B 93, 125204 (2016).

[77] S. Ozaki, M. Sasaki, and S. Adachi, Phys. Status Solidi A 203, 2648 (2006).

[78] G. V. Vajenine, C. Hoch, R. E. Dinnebier, A. Senyshyn, and R. Niewa, Z. Anorg. Allg. Chem. 636, 94 (2010).

[79] F. Kong, Y. Liu, B. Wang, Y. Wang, Y. Hu, L. Wang, and L. Tang, Physica B 407, 2272 (2012).
[80] CRC Handbook of Chemistry and Physics, 84th ed., edited by D. R. Lide (CRC Press, Boca Raton, 2004).

[81] Group IV Elements, IV-IV and III-V Compounds. Part B Electronic, Transport, Optical and other Properties, edited by O. Madelung, U. Rössler, and M. Schulz (Springer, Berlin, Heidelberg, 2002), pp. 1-6.

[82] W. Miller, C. W. Smith, D. S. Mackenzie, and K. E. Evans, J. Mater. Sci. 44, 5441 (2009).

[83] V. Heine, P. R. L. Welche, and M. T. Dove, J. Am. Ceram. Soc. 82, 1793 (1999).

[84] C. C. Meredith, J. W. Moberly, and M. Barlow, J. Less-Common Met. 18, 423 (1969).

[85] P. Gordon, J. Appl. Phys. 20, 908 (1949).

[86] C. A. Swenson, J. Appl. Phys. 70, 3046 (1991).

[87] W. Parrish and J. I. Langford, in International Tables for Crystallography Volume C: Mathematical, Physical and Chemical Tables, 2006 ed. (International Union of Crystallography, Dordrecht, 2006), Chap. 2.3.f1.1.6, pp. 42-79. 